\title{
Long-term Application of Novel Materials in Contaminated Soil: laboratory Testing for Metal(loid) Stabilisation Efficiency
}

\author{
Aikaterini Mitzia ${ }^{1}$, Martina Vítková ${ }^{1}$ \\ ${ }^{1}$ Faculty of Environmental Sciences, Czech University of Life Sciences Prague \\ Kamýcká 129, Praha - Suchdol, 165 00, Czech Republic \\ mitzia@fzp.czu.cz; vitkovam@fzp.czu.cz
}

\section{Extended Abstract}

The contamination of soils by risk metal(loid)s is persistent due to the non-degradable nature of such elements. Traditional remediation techniques, such as excavation of the contaminated soil and refilling with new soil, are costly and environmentally disruptive. Therefore, direct application of materials, which aim to immobilise risk elements in soil, is considered a viable remediation technique [1].

Considering that past mining and smelting areas remain a big environmental threat even if the polluting activities have ceased [2], new innovative approaches and materials towards soil remediation have been developed during the last years. One of the most popular products used in water (and soil remediation) is nano zero-valent iron (nZVI), which might come as pure nZVI or in numerous modifications [3]. Biochar (BC), a material coming from the pyrolysis of organic matter, has been traditionally used as a soil fertilizer while lately its application for soil remediation has been emerging [4]. The combination of nZVI with $\mathrm{BC}$ has been lately considered as an effective solution towards soil remediation, while at the same time limiting the disadvantages of pure nZVI (i.e., particle aggregation) [5].

In our study, i) a commercial product of nZVI, ii) a woody BC, and iii) a composite material synthesized by $40 \%$ of nZVI and $60 \%$ of biochar, were used. The tested materials were applied at a ratio of $2 \% \mathrm{w} / \mathrm{w}$ in a heavily contaminated soil originating from a post-mining area in the Czech Republic. The main contaminants were $\mathrm{Zn}\left(3.01 \mathrm{~g} \mathrm{~kg}^{-1}\right)$ and $\mathrm{Pb}(3.85 \mathrm{~g}$ $\left.\mathrm{kg}^{-1}\right)$. The application of the materials into the soil was followed by a 1-year incubation period, during which the samples were maintained at a certain moisture level. Various types of soil extractions were implemented at the end of the incubation period in order to test the metal stabilisation efficiency of the used materials.

The purpose of this study was to investigate the long-term potentials of soil remediation using innovative materials such as pure BC, nZVI including its oxidation products and an nZVI/BC modification. According to the results, all of the tested products are promising materials towards soil remediation from risk metal(loid)s in the long-term. Significant immobilisation of the target contaminants was determined in the amended soil samples. At the same time, the monitored physico-chemical properties were assumed to be the key factors affecting the solids' behaviour.

Extended studies regarding metal(loid) stabilisation in soils are still scarce. However, evaluation of the long-term behaviour of novel materials used in soil remediation is necessary before they can be safely applied in the field-scale.

The present research was funded by the Czech Science Foundation - project No. 18-24782Y and by the IGA of the Faculty of Environmental Sciences CULS Prague "Characterisation and testing of novel composites for metal/metalloid stabilisation in contaminated soils - No.20184227”.

\section{References}

[1] Khalid, S., Shahid, M., Niazi, N. K., Murtaza, B., Bibi, I., \& Dumat, C. "A comparison of technologies for remediation of heavy metal contaminated soils". J. Geochem. Explor. vol. 182, pp. 247-268, 2017.

[2] Ettler, V. "Soil contamination near non-ferrous metal smelters: a review". Appl. Geochem., vol. 64, pp. 56-74, 2016.

[3] Stefaniuk, M., Oleszczuk, P., \& Ok, Y. S. "Review on nano zerovalent iron (nZVI): from synthesis to environmental applications". Chem. Eng. J., vol. 287, pp. 618-632, 2016.

[4] Anawar, H. M., Akter, F., Solaiman, Z. M., \& Strezov, V. "Biochar: an emerging panacea for remediation of soil contaminants from mining, industry and sewage wastes". Pedosphere, vol. 25, no. 5, pp. 654-665, 2015. 
[5] Wang, S., Zhao, M., Zhou, M., Li, Y.C., Wang, J., Gao, B., Sato, S., Feng, K., Yin, W., Igalavithana, A.D. \& Oleszczuk, P. "Biochar-supported nZVI (nZVI/BC) for contaminant removal from soil and water: a critical review". $J$. Hazard. Mater. vol. 373, pp.820-834,2019. 